\title{
Pharmacophore Modeling, Virtual Screening and Molecular Docking Studies for Identification of New Inverse Agonists of Human Histamine $\mathrm{H}_{1}$ Receptor
}

\author{
Sundarapandian Thangapandian, Navaneethakrishnan Krishnamoorthy, Shalini John, Sugunadevi Sakkiah, \\ Prettina Lazar, Yuno Lee, and Keun Woo Lee*
}

\author{
Division of Applied Life Science (BK21 Program), Environmental Biotechnology National Core Research Center \\ (EB-NCRC), Plant Molecular Biology and Biotechnology Research Center (PMBBRC), \\ Gyeongsang National University, Jinju 660-701, Korea. *E-mail: kwlee@gnu.ac.kr. \\ Received October 6, 2009, Accepted December 7, 2009
}

\begin{abstract}
Human histamine $\mathrm{H}_{1}$ receptor (HHR1) is a $\mathrm{G}$ protein-coupled receptor and a primary target for antiallergic therapy. Here, the ligand-based three-dimensional pharmacophore models were built from a set of known HHR1 inverse agonists using HypoGen module of CATALYST software. All ten generated pharmacophore models consist of five essential features: hydrogen bond acceptor, ring aromatic, positive ionizable and two hydrophobic functions. Best model had a correlation coefficient of 0.854 for training set compounds and it was validated with an external test set with a high correlation value of 0.925 . Using this model Maybridge database containing 60,000 compounds was screened for potential leads. A rigorous screening for drug-like compounds unveiled RH01692 and SPB00834, two novel molecules for HHR1 with good CATALYST fit and estimated activity values. The new lead molecules were docked into the active site of constructed HHR1 homology model based on recently crystallized squid rhodopsin as template. Both the hit compounds were found to have critical interactions with Glu177, Phe432 and other important amino acids. The interpretations of this study may effectively be deployed in designing of novel HHR1 inverse agonists.
\end{abstract}

Key Words: Pharmacophore, Inverse agonists, Histamine H1 receptor, Lipinski’s rule, LigandFit

\section{Introduction}

Human histamine $\mathrm{H}_{1}$ receptor (HHR1) is one of the four histamine receptors namely $\mathrm{H}_{1}, \mathrm{H}_{2}, \mathrm{H}_{3}$ and $\mathrm{H}_{4}$. These receptors involved in a variety of physiological actions such as inflammation, gastric acid secretion, neurotransmitter release and mast cell mediated chemotaxis upon binding to the biogenic amine called histamine. ${ }^{1,2}$ HHR 1 belongs to class I of the G proteincoupled receptors (GPCRs) and it interacts with $\mathrm{G}$ proteins to activate phospholipase $\mathrm{C}^{3} \mathrm{HHR} 1$ is known to be involved in various inflammatory effects like smooth muscle contraction, negative inotropism and depolarization etc. GPCRs constitute the largest family of cell surface proteins involved in signal transduction. ${ }^{4,5}$ Intrinsically, they are the major targets for the antiallergic drug therapy due to the role of HHR1 in allergic reactions. Among the examples of available medicines interacting with GPCRs antihypertensive beta-blockers, opioid receptor agonists such as morphine, histamine $\mathrm{H}_{1}$ and $\mathrm{H}_{2}$ receptor antagonists as anti-allergic agents and antacids, respectively, and anti-depressants such as clozapine have gained their clinical importance. ${ }^{6}$

It is believed that GPCRs activate their associated signal transduction pathways not only upon agonist activation but also in the absence of agonists, resulting in constitutive receptor activity. Such activity may be calmed down by so-called inverse agonists that were originally classified as antagonists. The concept of constitutively active GPCRs is firmly rooted in receptor pharmacology. In order to explain all these one has to understand a receptor that can exist in more than one state, one active $\left(\mathrm{R}^{*}\right)$ and other inactive (R). Agonists and some drugs prefer the active state $\mathrm{R}^{*}$, while inverse agonists go for $\mathrm{R}$. ${ }^{7}$ In this concept 'neutral antagonists' would not alter the $\mathrm{R} / \mathrm{R} *$ distribution. Various human diseases have been developed as results of constitutive receptor activity. ${ }^{8}$ It is evident that inverse agonists are essential for the treatment of these diseases. ${ }^{9}$ Even well known HHR1 antagonists such as mepyramine, acrivastine, cetirizine, epinastine, loratadine are inverse agonists. ${ }^{8}$ Although an inverse HHR1 agonist would suppress any apparent constitutive HHR1 activity, long term exposure of cells expressing constitutively active GPCRs to inverse agonists may result in receptor up-regulation. The development of novel inverse HHR1 agonists would give a pharmacological tool to study the potential physiological role of constitutive HHR1 activity which is not yet clear.

In this study, we focused to develop a valid pharmacophore model for HHR1 inverse agonists, using it in virtual screening for new lead compounds and find their interactions with catalytic residues of HHR 1 by molecular docking. We have applied pharmacophore modeling and various molecular modeling methodologies to achieve this goal. Our study revealed the pharmacophore features that are essential for a HHR 1 inverse agonist and led us to identify the new molecules with the greater affinity for the HHR1. Our results were also proved with molecular docking study.

\section{Methods}

Pharmacophore model generation using HypoGen. Pharmacophore modeling calculations were carried out using CATALYST 4.11 (Accelrys, San Diego, USA). ${ }^{10}$ Thirty of thirty six diverse ligands, which are known for their HHR1 affinity, were taken as training set to develop the pharmacophore model as 
Table 1. Training and test set molecules

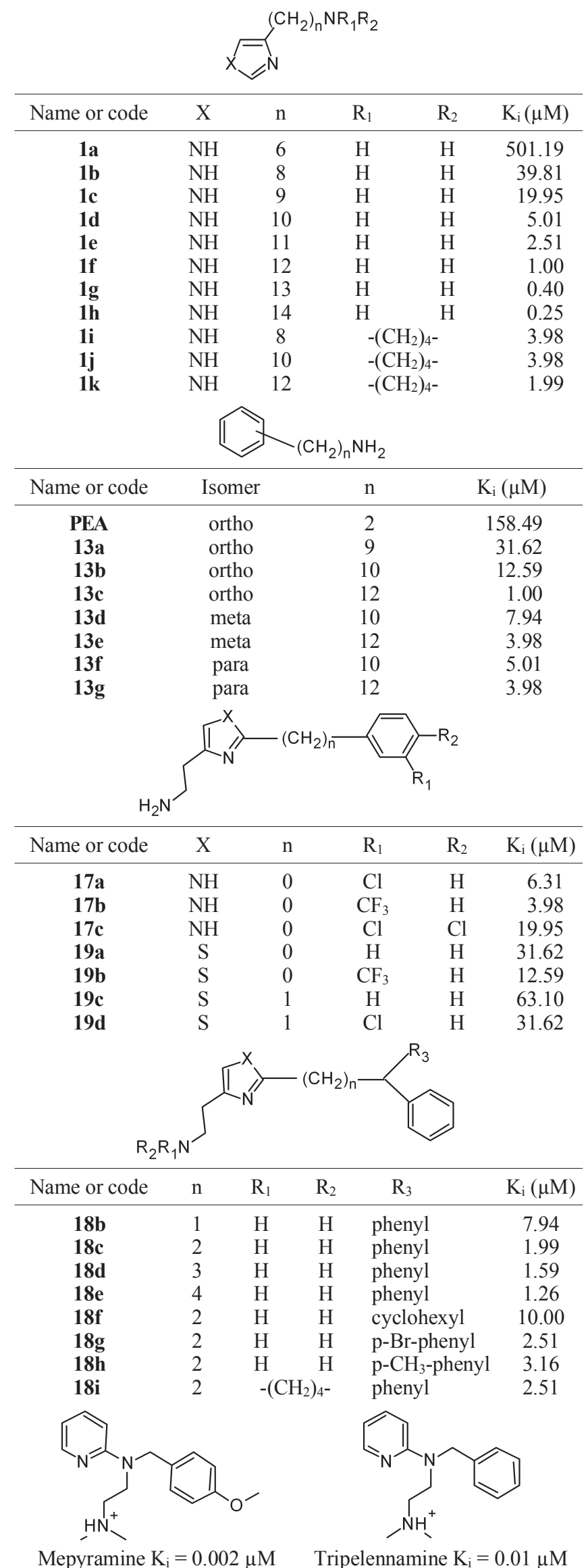

their $\mathrm{K}_{\mathrm{i}}$ values range from $0.002 \mu \mathrm{M}$ to $501.18 \mu \mathrm{M}$ over five orders of magnitude (Table 1). ${ }^{8}$ Six compounds named PEA, 1c, $18 b, 13 e, 18 i$ and $13 c$ were selected to be an external test set, which is used in the validation study of the generated pharmacophore models. All compounds in the training set were built using the $2 \mathrm{D} / 3 \mathrm{D}$ visualizer in CATALYST and minimized to the closest local minimum based on a modified CHARMmlike force field ${ }^{11}$ implemented within the Confirm module in CATALYST. CATALYST generated a group of representative conformational models for each compound in the training set using the Poling algorithm and the best conformational analysis method. ${ }^{12-14}$ Poling explicitly promotes conformational variation by forcing similar conformers away from each other. Every training set member is comprised of a collection of conformers that covers the conformational space accessible to the molecule within a given energy range. Diverse conformational models for each compound were generated using an energy constraint of $20 \mathrm{kcal} / \mathrm{mol}$ and 250 as the maximum number of conformers. All other parameters used were the default values.

HypoGen module implemented in CATALYST was used for pharmacophore generation. The uncertainty factor for each compound represents the range of uncertainty in the activity value based on the expected statistical sprawling of biological data collection. Here, this factor was defined as the default value of 3. Mepyramine was mentioned as a 'reference compound' specifying a 'Principal' value of 2 . The 'Principal' value and 'MaxOmitFeat' values were set to 0 for all other compounds in the training set. Pharmacophore models were then generated and the top 10 scoring hypotheses were exported for further calculations. The activity value of each training set compound is estimated using regression parameters. The relationship between geometric fit value and the activity value is utilized for this computation.

Pharmacophore model validation and database searching. In order to test whether the models identify active compounds and predict their activity accurately, we have used an external test set of compounds to evaluate the predictive ability of the best pharmacophore model. ${ }^{15,16}$ The test set included 6 compounds which are experimentally known for their HHR1 affinity. The pharmacophore model which scored a top CATALYST 'Maximum Fit' value, good cost difference and predicted the test set compounds with a high correlation was used as a query to screen the Maybridge (Maybridge Co. Ltd., UK) chemical database consisting of 60,000 structurally diverse small molecules. All queries were performed using the Best Flexible search databases/Spreadsheet method. A molecule must fit all the features of the pharmacophore to be retrieved as a hit. Discovery Studio 2.0 (DS) (Accelrys Co. Ltd., San Diego, USA) was used in further screening to identify the drug-like molecules by applying various filters. Novelty of the final hit compounds was ensured using Scifinder Scholar search (https://scifinder.cas.org) and Pubchem bioactivity analysis (http://pubchem.ncbi.nlm.nih. gov/).

Homology modeling. Homology model for HHR1 was built using SWISS-MODEL program, ${ }^{17-21}$ an online automated homology modeling server. Recently determined squid rhodopsin structure (PDB ID: 2Z73) was used as a template. The output model was validated using PROCHECK and the manual in- 
Table 2. Statistical information of top 10 pharmacophore hypotheses generated by HypoGen program

\begin{tabular}{cccccccc}
\hline \multirow{2}{*}{ Hypo no. } & \multirow{2}{*}{ Features } & \multirow{2}{*}{ Maximum Fit } & \multicolumn{2}{c}{ Training set } & \multicolumn{2}{c}{ Test set } \\
\cline { 4 - 7 } \cline { 5 - 7 } & & & Total cost & $\Delta$ cost & RMSD $(\AA)$ & Correlation (r) & Correlation $(\mathrm{r})$ \\
\hline 1 & HHARP & 11.291 & 125.714 & 44.770 & 1.043 & 0.875 & 0.916 \\
2 & HHARP & 11.464 & 127.713 & 42.771 & 1.018 & 0.860 & 0.626 \\
3 & HHARP & 11.763 & 128.040 & 42.444 & 1.105 & 0.859 & 0.564 \\
4 & HHARP & 10.842 & 128.597 & 41.887 & 1.138 & 0.849 & 0.924 \\
5 & HHARP & 12.144 & 129.056 & 41.428 & 1.126 & 0.854 & 0.925 \\
6 & HHARP & 10.703 & 129.290 & 41.194 & 1.159 & 0.843 & 0.724 \\
7 & HHARP & 9.385 & 132.879 & 37.605 & 1.259 & 0.812 & 0.832 \\
8 & HHARP & 10.191 & 133.105 & 37.379 & 1.267 & 0.809 & 0.918 \\
9 & HHARP & 8.946 & 133.773 & 36.711 & 1.278 & 0.805 & 0.558 \\
10 & HHARP & 10.379 & 134.405 & 36.079 & 1.299 & 0.797 & 0.848 \\
\hline
\end{tabular}

$\Delta \operatorname{cost}($ null cost - total cost); null cost $=170.484$; fixed cost $=109.033$; configuration cost $=7$; all cost values are in bits; $H, A, R$ and $\mathrm{P}$ represent Hydrophobic, Hydrogen bond acceptor, Ring aromatic and Positive ionizable group respectively.

vestigation of transmembrane (TM) regions. $^{22}$

Molecular docking. LigandFit, a modern docking program within DS, was used for the docking calculations. Protein coordinates for docking were taken from the homology modeled structure of HHR1. The LigandFit docking procedure consists of two parts: a) cavity detection to identify and select the region of the protein as the active site for docking and $b$ ) docking ligands to a selected site. ${ }^{23} 3 \mathrm{D}$ regular grids of points are employed for site detection and also for estimating the interaction energy of the ligand with the protein during the docking. All calculations were performed with the set of default parameters. The top 25 docked conformations were allowed to be saved. Docking score was evaluated for the 25 saved ligand conformations using a set of scoring functions as implemented in DS program including LigScore1, LigScore2, PLP1, PLP2 and PMF. Consensus scoring function had been used to evaluate and rank the ligand binding affinities.

\section{Results and Discussion}

Pharmacophore modeling. Ten hypotheses were produced by CATALYST using a training set of 30 compounds (Table 2). All the generated pharmacophore models possessed the following five chemical features: hydrogen bond acceptor (HBA), ring aromatic (RA), positive ionizable (PI) and two hydrophobic (HP) functions. This means that the five chemical features could effectively map the chemical features of the training set compounds and it is in line with the information that was reported recently. ${ }^{24}$ In general, the distance between the PI function (the basic nitrogen atom) and aromatic part of the ligand is expected to be around $6 \AA .^{24}$ In our model, it is $4.2 \AA$ between PI and RA, $6.06 \AA$ and $6.98 \AA$ between PI and two HP features, respectively. These observations support our pharmacophore model for its reasonable spatial arrangements. A significant hypothesis must possess the large difference between null and fixed cost values. ${ }^{25}$ In this study, the null cost value of the top 10 hypotheses is 170.484, and the fixed cost value is 109.033. Configuration cost value is 7 . In simple terms, there should be a large difference between fixed cost and null cost with a value of $40-60$ bits for the unit of cost, which would imply a $75-90 \%$ pro- bability for correlating the experimental and estimated activity data. The total cost of any hypothesis should be close to the fixed cost for a good model. In our study, all ten hypotheses have a total cost close to the fixed cost value. The difference between the fixed cost and null cost is 61.451 bits and may lead to a meaningful pharmacophore model. The cost difference between all ten hypotheses and the null hypothesis varies between 45 and 36 bits with a low cost range, 9 bits, between the first and tenth hypothesis. However, the first six hypotheses have the cost difference more than 40 . Therefore, we speculate that $75-90 \%$ possibility of representing a true correlation in the experimental and estimated activity data for these hypotheses. Table 2 shows these statistical parameters and predicting power of all pharmacophore models. The root mean square deviation (RMSD) indicates the quality of 'prediction' for the training set. The RMSD of all ten hypotheses ranged from $1.043 \AA$ to $1.299 \AA$. Besides this cost analysis, the most obvious method to validate the hypotheses is testing the ability to predict the activity of the training set compounds. In our case the fifth hypothesis (Hypo5) was found to have a maximum fit value for the training set compounds and the virtue of it is confirmed with their low error values for most active compounds (see table $\mathrm{S} 1$ in supplementary material). Maximum fit value indicates the overall fitness of all the training set compounds on a particular pharmacophore during pharmacophore generation. All first six hypotheses have scored well in terms of cost difference, RMSD and correlation values. However, Hypo5 scored a higher 'Maximum fit' value and also predicted the test set with a high correlation coefficient $\left(\mathrm{r}^{2}=0.925\right)$ consisting small deviation in terms of cost difference, RMSD and correlation values to that of first four hypotheses. Thus Hypo5 was considered as best hypothesis and denoted as HypoBest in further text and shown in Figure 1 with its inter feature distances. Table 2 lists the maximum fit values of all ten hypotheses. HypoBest predicted most of the activity values considerably for the training set compounds except for the least active compound 1a, with the correlation value (r) of 0.854 . Figure 2 shows the HypoBest model and its overlay with most and least active compounds of the training set.

Figure 2A depicts one of the most active conformations of 


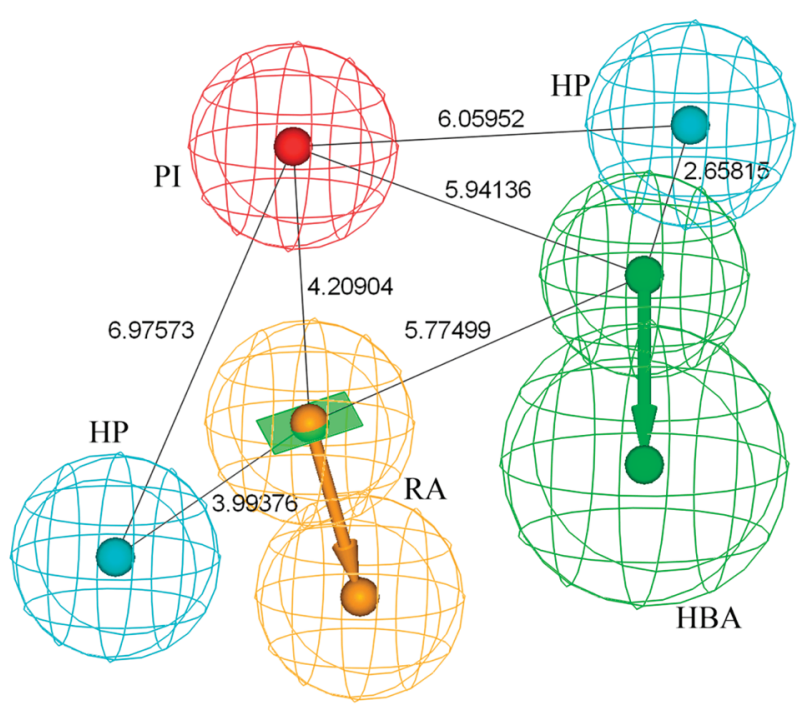

Figure 1. HypoBest model consists of a HBA (green), a RA (brown), a PI (red) and two HP (cyan) features.
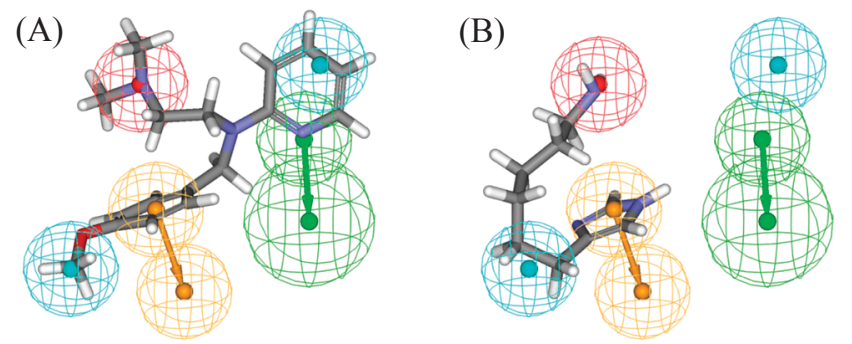

Figure 2. Top scoring model HypoBest aligned to mepyramine $\left(\mathrm{K}_{\mathrm{i}}=\right.$ $0.002 \mu \mathrm{M})(\mathrm{A})$ and compound $1 \mathrm{a}\left(\mathrm{K}_{\mathrm{i}}=501.19 \mu \mathrm{M}\right)(\mathrm{B})$ of training set. Pharmacophore features are colored as cyan for HP, red for PI, green for HBA and orange for RA.

mepyramine mapped onto HypoBest. Mepyramine is well aligned with all the features of the phamacophore model (HypoBest). Phenyl and pyridine rings overlap with the RA and one of two HP features while the methoxy, dimethyl amino groups and nitrogen of the pyridine ring overlap with second HP, PI and HBA features, respectively. Thus, the most active compound mepyramine in the training set maps well with the statistically most significant hypothesis, and the predicted activity of mepyramine toward HypoBest is well estimated. It is reported that the PI group of mepyramine interacts with the important Asp107 residue in the active site. ${ }^{26}$ Figure 2B shows the mapping of the training set compound 1a, for which the imidazole ring acts as RA feature. Long alkyl chain and its terminal amino group serve as HP and PI features, respectively, whereas the HBA and the other hydrophobic functionalities are missing.

The predictive power of HypoBest was analyzed using an external test set containing six compounds with a high range of activity. Structure and activity data of test set compounds [PEA, 1c, 13c, 13e, 18b \& 18i] are shown in Table 1. We have used Best Fit option of Compare/Fit module implemented in CATALYST for this procedure. The best conformation for every test set compound was generated and mapped upon the HypoBest

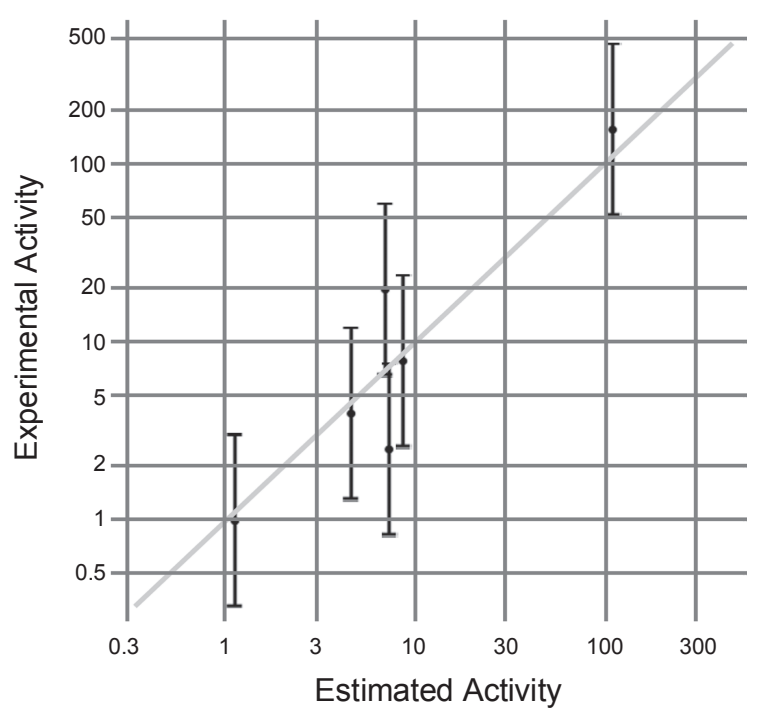

Figure 3. Correlation between experimental and estimated activity for the test set compounds. Correlation $(r)=0.925$.

Table 3. Test set compounds listed with their experimental, estimated activities and error values

\begin{tabular}{cccc}
\hline Compound & $\begin{array}{c}\text { Experimental activity } \\
\mathrm{K}_{\mathrm{i}}(\mu \mathrm{M})\end{array}$ & $\begin{array}{c}\text { Estimated activity } \\
\mathrm{K}_{\mathrm{i}}(\mu \mathrm{M})\end{array}$ & Error $^{a}$ \\
\hline PEA & 158.489 & 110.0 & -1.4 \\
$\mathbf{1 c}$ & 19.953 & 7.0 & -2.9 \\
$\mathbf{1 3 c}$ & 1.000 & 1.1 & 1.1 \\
$\mathbf{1 3 e}$ & 3.961 & 4.6 & 1.2 \\
$\mathbf{1 8 b}$ & 7.943 & 8.6 & 1.1 \\
$\mathbf{1 8 i}$ & 2.512 & 7.3 & 2.9 \\
\hline
\end{tabular}

${ }^{a}$ Positive value indicates that the estimated $\mathrm{K}_{\mathrm{i}}$ is higher than the experimental $\mathrm{K}_{\mathrm{i}}$; negative value indicates that the estimated $\mathrm{K}_{\mathrm{i}}$ is lower than the experimental $\mathrm{K}_{\mathrm{i}}$.

model to predict the fit and estimated activity values. Estimated activity values of members of test set were predicted well to their experimental activity with low error values and the result is reported in Table 3. Furthermore, HypoBest was used to perform a regression analysis with the test set compounds in order to check the predictive power of this model. Linear regression of the estimated activities for test set inhibitors versus the experimental ones showed a correlation value of 0.925 (Fig. 3 ). This result supports the validity of the statistically significant HypoGen hypothesis in predicting the affinity for HHR 1. Only two compounds with the activity range of $0.002-0.1 \mu \mathrm{M}$ have been included in the training set. Thus, the equal distribution of test compounds with activity could not be obtained in the particular range. The validated pharmacophore model could be used to perform virtual screening as a powerful tool to retrieve new potent molecules for designing HHR1 inverse agonists. In our study, the validated pharmacophore model was used to search Maybridge database consisting of 60,000 compounds. The query returned 3357 hits and they were all retrieved and subjected to screening by applying Lipinski rule of five, ${ }^{27}$ ADME (Absorption, Distribution, Metabolism and Excretion) properties such as human intestinal absorption, aque- 


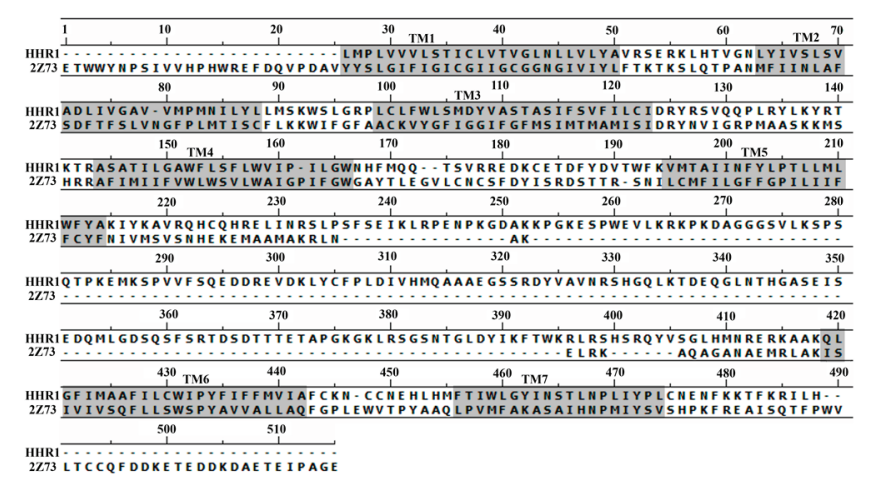

Figure 4. Sequence alignment between target (HHR1) and template protein (Squid rhodopsin; PDB ID: 2Z73). TM regions are in gray.
(A)

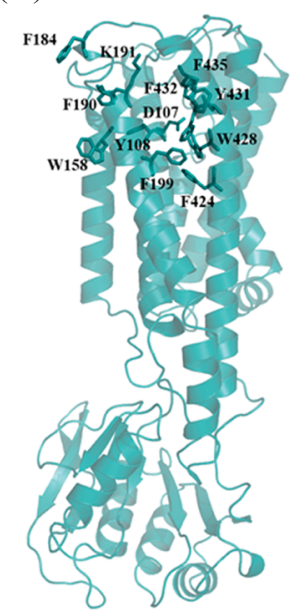

(B)

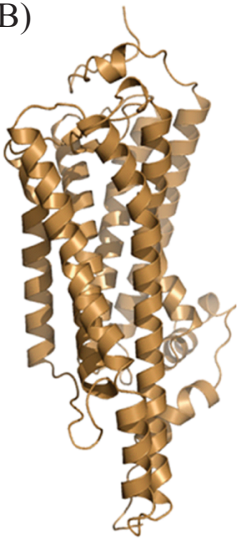

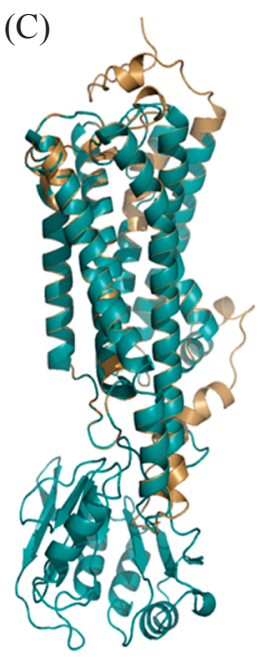

Figure 5. Homology modeling of HHR1 structure. (A) Homology model structure of HHR1 (cyan) with amino acids in the binding site; (B) Structure of template protein, squid rhodopsin (brown); (C) Overlay of HHR1 model and template protein. All structures are shown in ribbon representation.

ous solubility and blood brain barrier penetration. Toxicity prediction was also done in DS using hepatotoxic and CYP450 2D6 inhibition models. ${ }^{28}$ This screening was used to filter in only the compounds with drug-like properties. The 72 molecules were obtained from this rigorous screening. Finally only four molecules were obtained as hits from the second screening by restricting the minimum estimated activity to $0.1 \mu \mathrm{M}$. The four hit compounds were then subjected to docking study in order to verify their affinity towards HHR1. Since the crystal structure of HHR1 is not available we have constructed the model structure by homology modeling method. Several scoring functions available in DS 2.0 were used to evaluate and rank the each conformation of 4 hit compounds for their HHR1 binding affinities.

Homology modeling of HHR1 structure. Structure modeling of HHR 1 has been a great concern since the first crystal structure of GPCR was determined. Many research groups have obtained homology models for HHR1. ${ }^{26,29}$ In the beginning, it was bacterial rhodopsin used as template for the modeling followed by bovine rhodopsin (PDB ID: 1F88, $2.8 \AA$ resolu- tion). Here, high resolution structure of squid rhodopsin (PDB ID: $2 Z 73,2.5 \AA$ resolution) was used as template for the first time. Despite the controversies that exist on the validity of the GPCR models derived using rhodopsin structures as template, they have proved their applications on few drug-receptor interactions. $^{30}$ The sequence alignment of the HHR1 with squid rhodopsin is shown in Figure 4. Automated modeling mode of online comparative modeling server SWISS-MODEL was used to model the target structure. It showed about $21.5 \%$ identity, $45.9 \%$ similarity in the TM regions between HHR1 and squid rhodopsin sequences. Though the target sequence shared a high similarity with the TM regions of the template, there was no equivalence for its intracellular $\mathrm{G}$ protein binding region from the template. This region shows the long gap in the alignment from the residue 236 to 395 . It is reported that this region is involved in the binding of $\mathrm{G}$ protein. ${ }^{29}$ This long gap had no effect over the TM regions. The alignment was carefully investigated specially in TM regions for their integrity and we found that all of the critical structural elements known to be involved in the binding of its natural substrate, histamine, are intact. In addition to this the PROCHECK analysis was performed to validate the reliability of the model structure. It predicted $98.5 \%$ (86.2\% of residues in most favored regions, $10.9 \%$ of residues in additional allowed regions and $1.5 \%$ of residues in generously allowed regions) of residues of the model structure present in the allowed regions. Crystal structure of squid rhodopsin (PDB ID: 2Z73) had no residue in the disallowed regions and showed a high quality to be a template. Till date, no model for HHR1 has been constructed with this accuracy of $86.2 \%$ in most favoured regions using any rhodopsin as a template. Thus, our validation suggests that the backbone conformations of our model to be nearly as good as those of the template and could be a reliable model for further molecular docking study. Constructed model and its overlay upon the template protein are shown in Figure 5.

Molecular docking. We have docked histamine into the binding site and found its imidazole ring located within the liphophilic interaction range of Trp158 and far from Lys 191 . $^{26}$ However, we could not extract any information about the agonist binding from this model since it was built based on the inactive conformation of squid rhodopsin. Inverse agonists are the class of compounds that prefers the inactive conformation of the receptor. Therefore, this model could be used in the docking analyses of inverse agonists. Trp158, Phe432, and Phe435 are arranged in favorable positions to form a lipophilic cavity and take part in antagonist binding. ${ }^{28}$ The other aromatic residues, which are potential lipophilic interaction points, were found in TM3 (Tyr108), at the end of EC3 (Phe184), in TM5 (Phe190, Phe199) and in TM6 (Phe424, Trp428, Tyr431). Lys191 in TM5 was found in the internal side of the receptor, and seemed to be able to create an ionic interaction with the carboxylate group of zwitterionic inhibitors. ${ }^{26}$ We kept all the residues in mind while creating the binding site for the docking process.

All docking calculations were performed using LigandFit module as implemented in DS 2.0. The structural observation of the binding site of model suggested that one side of the active site of the receptor formed by a set of aromatic amino acids (F424, W428, Y431, F432 \& F435) making it highly 
Table 4. Comparison of dock scores between hit and known inhibitor molecules

\begin{tabular}{lcccccc}
\hline \multirow{2}{*}{ Compound } & \multicolumn{7}{c}{ Dock Scores } \\
\cline { 2 - 7 } & -LigScore1 & LigScore2 & -PLP1 & -PLP2 & -PMF & Consensus \\
\hline Mepyramine & 0.41 & 0.67 & 126.41 & 126.39 & 186.25 & 6 \\
Acrivastine & 8.51 & -13.82 & 121.12 & 126.85 & 178.16 & 5 \\
RH01692 & 0.47 & -1.98 & 138.89 & 130.47 & 197.29 & 6 \\
SPB00834 & 0.88 & -1.5 & 122.49 & 125.71 & 182.44 & 6 \\
\hline
\end{tabular}

hydrophobic in nature while a blend of aromatic (Y108 \& W158), negatively charged (D107 \& E177) and polar uncharged (S111) amino acids form the other side. This arrangement of amino acids confirms that the inhibitor has to bind in such a way that its PI and aromatic groups positioned towards the negatively charged amino acid and hydrophobic part of the catalytic site, respectively. Our molecular docking studies resulted the same positioning of ligands in the active site. Various cavities which can accommodate the ligand conformation were listed by LigandFit. The one formed by the above mentioned active site amino acids was chosen to dock the ligands. Mepyramine from first generation, acrivastine from second generation antihistamines along with other training set compounds and four new hits retrieved from the Maybridge chemical database were subjected to docking study. Figure 6 shows the final hit compounds aligned to the HypoBest model. LigScore1, LigScore2, PLP1, PLP2, PMF and consensus scoring functions were calculated. The results are summarized in Table 4 with hits those had relatively better docking scores. Mepyramine, which binds to HHR1 with high affinity, has generated strong hydrogen
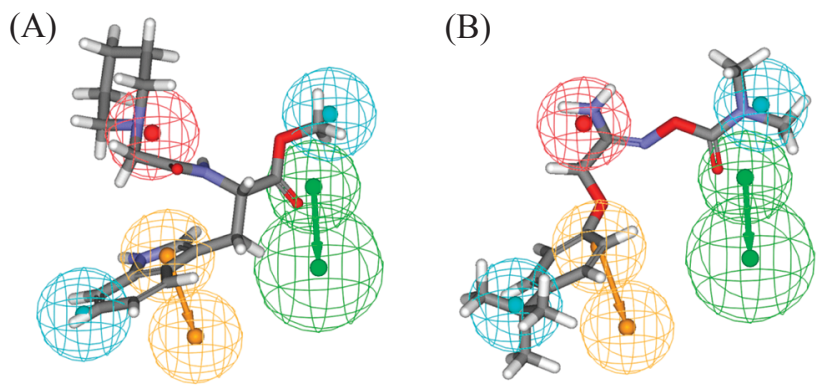

Figure 6. Overlay of hit compounds RH01692 (A) and SPB00834 (B) upon the HypoBest model. bond interactions with Asp107, Tyr108 and Glu177, and its two aryl groups were positioned in the lipophilic cavity, formed by other active site amino acids (Figure 7A). This orientation of mepyramine resembles the previously reported docking results by the other research group. ${ }^{26}$ Thus, these results provide the evidence that the active site remains intact during the homology modeling even though the different rhodopsin structures are used as templates. Acrivastine also has produced a similar type of orientation and the hydrogen bond interactions with the active site amino acids (Figure 7B). One of the hit compounds RH01692 has created the hydrogen bond interactions with the same set of amino acids and also with Phe 432 which is one of the aromatic residues in the active site (Figure 7C) and this suggests that it may act as an another critical interaction point for a ligand. Indole part of it oriented unto the hydrophobic part of the active site and makes it available for hydrophobic interactions. On the other hand, SPB00834 has also had its phenyl ring with $t$-butyl substitution towards the hydrophobic part of the active site but showed hydrogen bond interactions only with Glu177 and Phe432 not with Asp107 and Tyr108 and this could be the reason for its low dock score compared to RH01692.

Two compounds, RH01692 and SPB00834, out off four hits retrieved from the Maybridge database have shown excellent HypoGen estimated activity, fit values and docking consensus score. Figure 7 shows their docking conformations in the active site compared with the known active compounds mepyramine and acrivastine and Figure 8 shows $2 \mathrm{D}$ representation of their molecular structures. The estimated activity values, CATALYST fit values, consensus docking scores for RH01692 and SPB 00834 are $0.01 \mu \mathrm{M}$ and $0.03 \mu \mathrm{M}, 9.289$ and 9.029, 6 and 6 , respectively. RH01692 has scored equal to the tripelennamine, the second most active compound in the training set. RH01692 and SPB00834 have scored fit values close to the most active
(A)

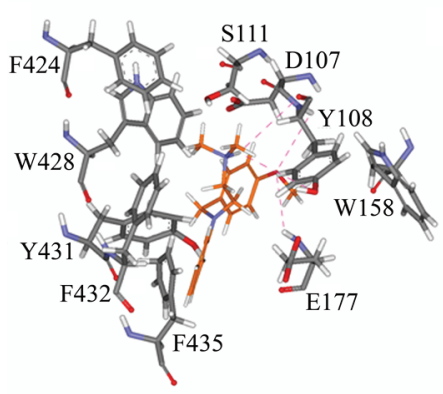

(B)

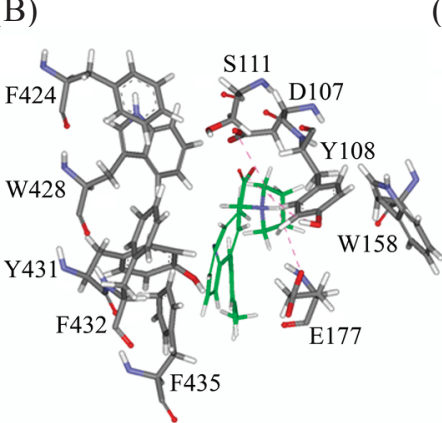

(C)

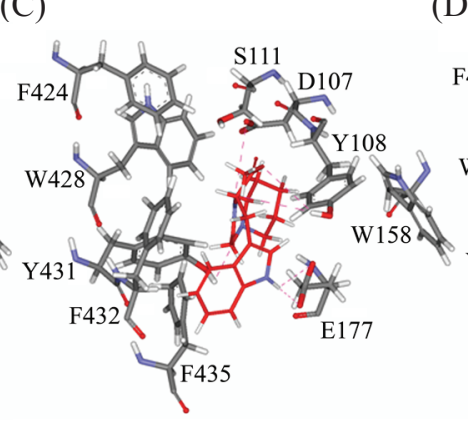

(D)

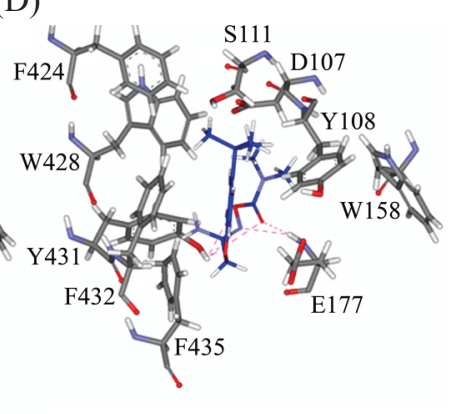

Figure 7. Molecular docking results. The docked ligands are shown with their hydrogen bond interactions: (A) Mepyramine (orange); (B) Acrivastine (green); (C) RH01692 (red); (D) SPB00834 (dark blue). Hydrogen bonds are shown in magenta as dotted lines. 


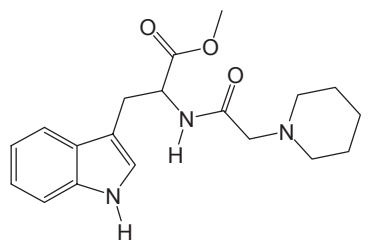

RH 01692

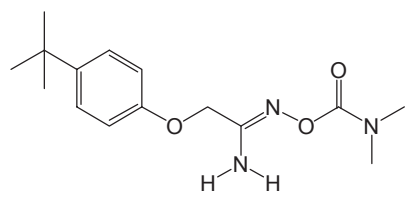

SPB 00834
Figure 8. 2D representation of final hit compounds.

compound in the training set and also have formed hydrogen bonds with critical amino acids. SciFinder Scholar search and pubchem bioactivity analysis confirmed that both hit compounds are novel and they have not been reported earlier for any antihistaminic activity. These results suggest that the hits obtained from this study can become superior leads in designing potent and novel HHR1 inverse agonists.

\section{Conclusion}

HHR1 is responsible for the various inflammatory effects of the monoamine, histamine. Pharmacophore and other molecular modeling techniques were applied in this study. The pharmacophore model HypoBest which was generated using training set of 30 compounds characterized by a high predictive ability. It was also proved on an external test set compounds with the correlation coefficient of 0.925 . HypoBest model was used as a $3 \mathrm{D}$ query to search a database to identify the compounds that possess the required features. The query was successful in retrieving compounds for HHR1 inhibition. All hit compounds were subjected to the rigorous filtering for compounds with drug-like properties. Docking of the final hit compounds on to the homology modeled structure of HHR1 revealed their affinity towards the receptor. Docking score, internal binding energy and how well they docked into HHR1 binding site, particularly their interactions with important amino acids of HHR 1 binding site have also been considered in the investigation. Despite the broad research none of the indole based antihistaminic molecules have reached the market yet. Compound RH01692 is an indole based compound and novel substitutions on the structure would provide some way to have a handful of new leads. SPB 00834 had a phenyl ring with novel substitutions as of the history of antihistamines concern. These results suggest that HypoBest can be used in screening of other available chemical databases to find out more novel scaffolds to design potent HHR 1 inverse agonists. For the first time, HHR1 structure has been modeled using squid rhodopsin as template and effectively used in docking studies to identify the key interacting residues. This HHR1 model can reliably be used in structure based virtual screening for novel scaffolds those can effectively bind in the active site.

Acknowledgments. All students were supported by a scholarship from the BK21 Program Programs by the Korea government (MEST). This work was also supported by grants from the MEST/NRF (National Research Foundation of Korea) to Environmental Biotechnology National Core Research Center (20090091489) and Pioneer Research Center Program (20090081539).

\section{References}

1. Steven, M. F.; Tom, I. B.; Richard, R. N.; Edward, M. R.; JeanPhillipe, P.; Anthony, P. D.; Michael, S.; Anthony, J. H. Pharmacol. Rev. 2005, 57, 279.

2. Hofstra, C. L.; Desai, P. J.; Thurmond, R. L.; Fung-Leung, W. P. J. Pharmacol. Exp. Ther. 2003, 305, 1212.

3. Hill, S. J.; Ganellin, C. R.; Timmerman, H.; Schwartz, J. C.; Shankley, N. P.; Young, J. M.; Schunack, W.; Levi, R.; Haas, H. L. Pharmacol. Rev. 1997, 49, 253.

4. Maria, J. M.; Silvio, G. J. TRENDS in Pharmacological Sciences 2001, 221, 368.

5. Louis, M. L. Mol. Biotechnol. 2008, 39, 239.

6. Willem, S.; Ineke van, W.; Adriaan, P. I. Med. Res. Rev. 2005, 25 , 398.

7. Prather, L. P. Sci. STKE. 2004, $215,1$.

8. Govoni, M.; Bakker, R. A.; Wetering, I.; Smit, J. M.; Menge, M. B. P.; Timmerman, H.; Elz, S.; Schunack, W.; Leurs, R. J. Med. Chem. 2003, 46, 5812.

9. Tao, Y. Pharmacol. Ther. 2008, 120, 129.

10. CATALYST 4.10 User Guide. 2005 Accelrys Inc., San Diego, CA, USA.

11. Brooks, B. R.; Brucolleri, R. E.; Olafson, B. D.; States, D. J.; Swaminathan, S.; Karplus, M. J. Comput. Chem. 1983, 4, 187.

12. Smellie, A.; Teig, S. L.; Towbin, P. J. Comput. Chem. 1995, 16, 171.

13. Smellie, A.; Kahn, S. D.; Teig, S. J. Chem. Inf. Comput. Sci. 1995, 35,285

14. Smellie, A.; Kahn, S. D.; Teig, S. J. Chem. Inf. Comput. Sci. 1995, 35,295 .

15. Sakkiah, S.; Krishnamoorthy, N.; Gajendrarao, P.; Thangapandian, S. Lee, Y.; Suh, J. K.; Kim, H. H.; Lee, K. W. Bull. Korean Chem. Soc. 2009, 30, 1152-1156.

16. Lee, Y.; Bharatham, N.; Bharatham, K.; Lee, K. W. Bull. Korean Chem. Soc. 2007, 28, 561-566.

17. Arnold, K.; Bordoli, L.; Kopp, J.; Schwede, T. Bioinformatics 2006, 22, 195.

18. Kopp, J.; Schwede, T. Nucleic Acids Research 2004, 32, D230.

19. Schwede, T.; Kopp, J.; Guex, N.; Peitsch, M. C. Nucleic Acids Research 2003, 31, 3381.

20. Guex, N.; Peitsch, M. C. Electrophoresis 1997, 18, 2714.

21. Peitsch, M. C. Nat. Biotechnol. 1995, 13, 658.

22. Laskowski, R. A.; MacArthur, M. W.; Moss, D.; Thornton, J. M. J. Appl. Cryst. 1993, 26, 283.

23. Venkatachalam, C. M.; Jiang, X.; Oldfield, T.; Waldman, M. J. Mol. Graph. Model 2003, 21, 289.

24. Anton, M. T.; Marc, J. D.; Hendrik, T.; Gabrielle, M. D. Quantitative Structure-Activity Relationships 2008, 11, 348.

25. Bharatham, N.; Bharatham, K.; Lee, K. W. J. Mol. Graph. Model 2007, 25, 813 .

26. Robert, K.; Zoltan, K.; Gyorgy, M. K. European J. Med. Chem. 2004, 39, 959.

27. Lipinski, C. A.; Lobbardo, F.; Dominy, B. W.; Feeny, P. J. Adv. Drug. Delivery Rev. 1997, 23, 3.

28. Venkatapathy, R.; Moudgal, C. J.; Bruce, R. M. J. Chem. Inf. Comput. Sci. 2004, 44, 1623.

29. Bissantz, C.; Bernard, P.; Hibert, M.; Rognan, D. Proteins 2003, 50,5 .

30. Kerstin, W.; Anton, M. T.; Martine, J. S.; Ronald, K.; Timmerman, H.; Rob, L. J. Biol. Chem. 1999, 274, 29994. 


\section{Pharmacophore Modeling, Virtual Screening and Molecular Docking Studies for Identification of New Inverse Agonists of Human Histamine $\mathrm{H}_{1}$ Receptor}

Sundarapandian Thangapandian, Navaneethakrishnan Krishnamoorthy, Shalini John, Sugunadevi Sakkiah, Prettina Lazar, Yuno Lee, and Keun Woo Lee*

Division of Applied Life Science (BK21 Program), Environmental Biotechnology National Core Research Center (EB-NCRC), Plant Molecular Biology and Biotechnology Research Center (PMBBRC),

Gyeongsang National University, Jinju 660-701, Korea.*E-mail: kwlee@gnu.ac.kr.

Received October 6, 2009, Accepted December 7, 2009

Key Words:

Table S1. Training set compounds with their fit, experimental, estimated and error values based on HypoBest.

\begin{tabular}{|c|c|c|c|c|}
\hline Compound & Fit & $\begin{array}{l}\text { Experimental activity } \\
\qquad\left(\mathrm{K}_{\mathrm{i}} \mu \mathrm{M}\right)\end{array}$ & $\begin{array}{l}\text { Estimated activity } \\
\qquad\left(\mathrm{K}_{\mathrm{i}} \mu \mathrm{M}\right)\end{array}$ & Error $^{a}$ \\
\hline Mepyramine & 10.39 & 0.002 & 0.001 & -1.5 \\
\hline Tripelennamine & 9.27 & 0.010 & 0.018 & 1.8 \\
\hline $1 \mathrm{~h}$ & 7.26 & 0.250 & 1.8 & 7.3 \\
\hline $1 \mathrm{~g}$ & 7.03 & 0.398 & 3.1 & 7.8 \\
\hline 1f & 7.10 & 1.000 & 2.6 & 2.6 \\
\hline $18 \mathrm{e}$ & 6.84 & 1.259 & 4.8 & 3.8 \\
\hline 18d & 6.70 & 1.585 & 6.7 & 4.2 \\
\hline $18 \mathrm{c}$ & 6.38 & 1.995 & 14 & 7 \\
\hline $1 \mathrm{k}$ & 7.15 & 1.995 & 2.3 & 1.2 \\
\hline $1 \mathrm{e}$ & 6.74 & 2.512 & 6.1 & 2.4 \\
\hline $18 \mathrm{~g}$ & 6.92 & 2.512 & 4 & 1.6 \\
\hline $18 \mathrm{~h}$ & 6.72 & 3.162 & 6.4 & 2 \\
\hline $\mathbf{1 i}$ & 6.80 & 3.981 & 5.2 & 1.3 \\
\hline $17 \mathrm{~b}$ & 6.73 & 3.981 & 6.2 & 1.6 \\
\hline $\mathbf{1 j}$ & 6.90 & 3.981 & 4.2 & 1 \\
\hline $13 \mathrm{~g}$ & 7.03 & 3.981 & 3.1 & -1.3 \\
\hline $13 \mathrm{f}$ & 6.73 & 5.012 & 6.2 & 1.2 \\
\hline 1d & 6.51 & 5.012 & 10 & 2.1 \\
\hline $17 \mathbf{a}$ & 6.66 & 6.310 & 7.3 & 1.2 \\
\hline 13d & 6.98 & 7.943 & 3.5 & -2.3 \\
\hline $18 f$ & 6.50 & 10.000 & 11 & 1.1 \\
\hline $19 b$ & 6.71 & 12.589 & 6.5 & -1.9 \\
\hline $13 b$ & 6.82 & 12.589 & 5.1 & -2.5 \\
\hline $17 \mathrm{c}$ & 6.82 & 19.953 & 5 & -4 \\
\hline $19 a$ & 6.62 & 31.623 & 7.9 & -4 \\
\hline 19d & 6.77 & 31.623 & 5.7 & -5.6 \\
\hline $13 a$ & 6.82 & 31.623 & 5.1 & -6.3 \\
\hline $1 b$ & 6.10 & 39.811 & 26 & -1.5 \\
\hline $19 \mathrm{c}$ & 6.19 & 63.096 & 21 & -3 \\
\hline $1 \mathrm{a}$ & 6.37 & 501.188 & 14 & -35 \\
\hline
\end{tabular}

${ }^{a}$ Positive value indicates that the estimated $\mathrm{K}_{\mathrm{i}}$ is higher than the experimental $\mathrm{K}_{\mathrm{i}}$; negative value indicates that the estimated $\mathrm{K}_{\mathrm{i}}$ is lower than the experimental $\mathrm{K}_{\mathrm{i}}$ 